\title{
On Hardy-type integral inequalities with the gamma function
}

\author{
Jianquan Liao* and Bicheng Yang
}

"Correspondence: Imath@163.com Department of Mathematics,

Guangdong University of

Education, Guangzhou, Guangdong 51003, P.R. China

\section{照 Springer}

\begin{abstract}
By means of real analysis and weight functions, we obtain a few equivalent conditions of two kinds of Hardy-type integral inequalities with the non-homogeneous kernel and parameters. The constant factors related to the gamma function are proved to be the best possible. We also consider the operator expressions and some cases of homogeneous kernel.
\end{abstract}

MSC: $26 \mathrm{D} 15$

Keywords: Hardy-type integral inequality; weight function; equivalent form; operator; norm

\section{Introduction}

If $0<\int_{0}^{\infty} f^{2}(x) d x<\infty$ and $0<\int_{0}^{\infty} g^{2}(y) d y<\infty$, then we have the following Hilbert's integral inequality (cf. [1]):

$$
\int_{0}^{\infty} \int_{0}^{\infty} \frac{f(x) g(y)}{x+y} d x d y<\pi\left(\int_{0}^{\infty} f^{2}(x) d x \int_{0}^{\infty} g^{2}(y) d y\right)^{\frac{1}{2}}
$$

where the constant factor $\pi$ is the best possible. In 1925, by introducing one pair of conjugate exponents $(p, q)$, Hardy [2] gave an extension of (1) as follows: For $p>1, \frac{1}{p}+\frac{1}{q}=1$, $f(x), g(y) \geq 0,0<\int_{0}^{\infty} f^{p}(x) d x<\infty$ and $0<\int_{0}^{\infty} g^{q}(y) d y<\infty$, we have

$$
\int_{0}^{\infty} \int_{0}^{\infty} \frac{f(x) g(y)}{x+y} d x d y<\frac{\pi}{\sin (\pi / p)}\left(\int_{0}^{\infty} f^{p}(x) d x\right)^{\frac{1}{p}}\left(\int_{0}^{\infty} g^{q}(y) d y\right)^{\frac{1}{q}},
$$

where the constant factor $\frac{\pi}{\sin (\pi / p)}$ is the best possible. Inequalities (1) and (2) are important in analysis and its applications (cf. [3, 4]).

In 1934, Hardy et al. gave an extension of (2) as follows: If $k_{1}(x, y)$ is a non-negative homogeneous function of degree $-1, k_{p}=\int_{0}^{\infty} k_{1}(u, 1) u^{\frac{-1}{p}} d u \in \mathbf{R}_{+}=(0, \infty)$, then

$$
\int_{0}^{\infty} \int_{0}^{\infty} k_{1}(x, y) f(x) g(y) d x d y<k_{p}\left(\int_{0}^{\infty} f^{p}(x) d x\right)^{\frac{1}{p}}\left(\int_{0}^{\infty} g^{q}(y) d y\right)^{\frac{1}{q}},
$$

where the constant factor $k_{p}$ is the best possible (cf. [3], Theorem 319). Additionally, a Hilbert-type integral inequality with the non-homogeneous kernel is proved as follows:

(c) The Author(s) 2017. This article is distributed under the terms of the Creative Commons Attribution 4.0 International License (http://creativecommons.org/licenses/by/4.0/), which permits unrestricted use, distribution, and reproduction in any medium, provided you give appropriate credit to the original author(s) and the source, provide a link to the Creative Commons license, and indicate if changes were made. 
If $h(u)>0, \phi(\sigma)=\int_{0}^{\infty} h(u) u^{\sigma-1} d u \in \mathbf{R}_{+}$, then

$$
\begin{aligned}
& \int_{0}^{\infty} \int_{0}^{\infty} h(x y) f(x) g(y) d x d y \\
& \quad<\phi\left(\frac{1}{p}\right)\left(\int_{0}^{\infty} x^{p-2} f^{p}(x) d x\right)^{\frac{1}{p}}\left(\int_{0}^{\infty} g^{q}(y) d y\right)^{\frac{1}{q}},
\end{aligned}
$$

where the constant factor $\phi\left(\frac{1}{p}\right)$ is still the best possible ( $c f$. [3], Theorem 350).

In 1998, by introducing an independent parameter $\lambda>0$, Yang gave a best extension of (1) with the kernel $\frac{1}{(x+y)^{\lambda}}(c f$. [5, 6]). In 2004, by introducing another pair conjugate exponents $(r, s)$, Yang [7] gave an extension of (2) as follows: If $\lambda>0, r>1, \frac{1}{r}+\frac{1}{s}=1, f(x)$, $g(y) \geq 0,0<\int_{0}^{\infty} x^{p\left(1-\frac{\lambda}{r}\right)-1} f^{p}(x) d x<\infty$ and $0<\int_{0}^{\infty} y^{q\left(1-\frac{\lambda}{s}\right)-1} g^{q}(y) d y<\infty$, then

$$
\begin{aligned}
& \int_{0}^{\infty} \int_{0}^{\infty} \frac{f(x) g(y)}{x^{\lambda}+y^{\lambda}} d x d y \\
& <\frac{\pi}{\lambda \sin (\pi / r)}\left(\int_{0}^{\infty} x^{p\left(1-\frac{\lambda}{r}\right)-1} f^{p}(x) d x\right)^{\frac{1}{p}}\left(\int_{0}^{\infty} y^{q\left(1-\frac{\lambda}{s}\right)-1} g^{q}(y) d y\right)^{\frac{1}{q}},
\end{aligned}
$$

where the constant factor $\frac{\pi}{\lambda \sin (\pi / r)}$ is the best possible. For $\lambda=0, r=q, s=p$, (5) reduces to (2); For $\lambda=1, r=p, s=q$, (5) reduces to the dual form of (2) as follows:

$$
\begin{aligned}
& \int_{0}^{\infty} \int_{0}^{\infty} \frac{f(x) g(y)}{x+y} d x d y \\
& \quad<\frac{\pi}{\sin (\pi / p)}\left(\int_{0}^{\infty} x^{p-2} f^{p}(x) d x\right)^{\frac{1}{p}}\left(\int_{0}^{\infty} y^{q-2} g^{q}(y) d y\right)^{\frac{1}{q}}
\end{aligned}
$$

For $p=q=2$, both (2) and (7) reduce to (1).

In 2005, in [8] one also gave an extension of (2) and (5) with the kernel $\frac{1}{(x+y)^{\lambda}}$. Krnić et al. [9-14] provided some extensions and particular cases of (2), (3) and (4) with parameters. In 2009, Yang gave an extension of (3) and (5) as follows (cf. [15, 16]): If $\lambda_{1}+\lambda_{2}=\lambda \in \mathbf{R}=$ $(-\infty, \infty), k_{\lambda}(x, y)$ is a non-negative homogeneous function of degree $-\lambda$, satisfying

$$
k_{\lambda}(u x, u y)=u^{-\lambda} k_{\lambda}(x, y) \quad(u, x, y>0)
$$

and

$$
k\left(\lambda_{1}\right)=\int_{0}^{\infty} k_{\lambda}(u, 1) u^{\lambda_{1}-1} d u \in \mathbf{R}_{+}
$$

then

$$
\begin{aligned}
& \int_{0}^{\infty} \int_{0}^{\infty} k_{\lambda}(x, y) f(x) g(y) d x d y \\
& \quad<k\left(\lambda_{1}\right)\left(\int_{0}^{\infty} x^{p\left(1-\lambda_{1}\right)-1} f^{p}(x) d x\right)^{\frac{1}{p}}\left(\int_{0}^{\infty} y^{q\left(1-\lambda_{2}\right)-1} g^{q}(y) d y\right)^{\frac{1}{q}},
\end{aligned}
$$


where the constant factor $k\left(\lambda_{1}\right)$ is the best possible. For $\lambda=1, \lambda_{1}=\frac{1}{q}, \lambda_{2}=\frac{1}{p}$, (7) reduces to (3). Additionally, an extension of (4) was given as follows:

$$
\begin{aligned}
& \int_{0}^{\infty} \int_{0}^{\infty} h(x y) f(x) g(y) d x d y \\
& \quad<\phi(\sigma)\left(\int_{0}^{\infty} x^{p(1-\sigma)-1} f^{p}(x) d x\right)^{\frac{1}{p}}\left(\int_{0}^{\infty} y^{q(1-\sigma)-1} g^{q}(y) d y\right)^{\frac{1}{q}},
\end{aligned}
$$

where the constant factor $\phi(\sigma)$ is the best possible ( $c f$. [17]). For $\sigma=\frac{1}{p}$, (8) reduces to (4). Some equivalent inequalities of (7) and (8) are considered by [16]. In 2013, Yang [17] studied the equivalency between (7) and (8). In 2017, Hong [18] studied an equivalent condition between (7) with a few parameters.

$\operatorname{Remark} 1$ (cf. [17]) If $h(x y)=0$, for $x y>1, \phi(\sigma)=\int_{0}^{1} h(u) u^{\sigma-1} d u=\phi_{1}(\sigma) \in \mathbf{R}_{+}$, then (8) reduces to the following Hardy-type integral inequality with the non-homogeneous kernel:

$$
\begin{aligned}
& \int_{0}^{\infty} g(y)\left(\int_{0}^{\frac{1}{y}} h(x y) f(x) d x\right) d y \\
& \quad<\phi_{1}(\sigma)\left(\int_{0}^{\infty} x^{p(1-\sigma)-1} f^{p}(x) d x\right)^{\frac{1}{p}}\left(\int_{0}^{\infty} y^{q(1-\sigma)-1} g^{q}(y) d y\right)^{\frac{1}{q}} ;
\end{aligned}
$$

if $h(x y)=0$, for $x y<1, \phi(\sigma)=\int_{1}^{\infty} h(u) u^{\sigma-1} d u=\phi_{2}(\sigma) \in \mathbf{R}_{+}$, then (8) reduces to the following another kind of Hardy-type integral inequality with the non-homogeneous kernel:

$$
\begin{aligned}
& \int_{0}^{\infty} g(y)\left(\int_{\frac{1}{y}}^{\infty} h(x y) f(x) d x\right) d y \\
& \quad<\phi_{2}(\sigma)\left(\int_{0}^{\infty} x^{p(1-\sigma)-1} f^{p}(x) d x\right)^{\frac{1}{p}}\left(\int_{0}^{\infty} y^{q(1-\sigma)-1} g^{q}(y) d y\right)^{\frac{1}{q}} .
\end{aligned}
$$

In this paper, by real analysis and the weight functions, we obtain a few equivalent conditions of two kinds of Hardy-type integral inequalities with the non-homogeneous kernel and parameters as $\frac{\left(\min \{x y, 1)^{\alpha}|\ln x y|^{\beta}\right.}{(\max \{x y, 1\})^{\lambda+\alpha}}$. The constant factors related to the gamma function are proved to be the best possible. We also consider the operator expressions and some cases of homogeneous kernel.

\section{Two lemmas}

For $\beta>-1, \sigma+\mu=\lambda \in \mathbf{R}$, we set

$$
h(u):=\frac{(\min \{u, 1\})^{\alpha}|\ln u|^{\beta}}{(\max \{u, 1\})^{\lambda+\alpha}} \quad(u>0) .
$$

Then, for $\sigma>-\alpha$, setting $v=-(\sigma+\alpha) \ln u$, we find

$$
\begin{aligned}
k_{1}(\sigma) & :=\int_{0}^{1} \frac{(\min \{u, 1\})^{\alpha}|\ln u|^{\beta}}{(\max \{u, 1\})^{\lambda+\alpha}} u^{\sigma-1} d u \\
& =\int_{0}^{1} u^{\sigma+\alpha-1}(-\ln u)^{\beta} d u=\frac{1}{(\sigma+\alpha)^{\beta+1}} \int_{0}^{\infty} v^{\beta} e^{-v} d v=\frac{\Gamma(\beta+1)}{(\sigma+\alpha)^{\beta+1}} \in \mathbf{R}_{+} .
\end{aligned}
$$


For $\mu>-\alpha$, we find

$$
\begin{aligned}
k_{2}(\sigma) & :=\int_{1}^{\infty} \frac{(\min \{u, 1\})^{\alpha}|\ln u|^{\beta}}{(\max \{u, 1\})^{\lambda+\alpha}} u^{\sigma-1} d u \\
& =\int_{0}^{1} v^{\mu+\alpha-1}(-\ln v)^{\beta} d v=\frac{\Gamma(\beta+1)}{(\mu+\alpha)^{\beta+1}}=k_{1}(\mu) \in \mathbf{R}_{+},
\end{aligned}
$$

where $\Gamma(\eta):=\int_{0}^{\infty} v^{\eta-1} e^{-v} d v(\eta>0)$ is the gamma function $(c f .[19])$.

Lemma 1 If $p>1, \frac{1}{p}+\frac{1}{q}=1, \sigma_{1} \in R, \beta>-1, \sigma>-\alpha$, there exists a constant $M_{1}$, such that, for any non-negative measurable functions $f(x)$ and $g(y)$ in $(0, \infty)$, the following inequality:

$$
\begin{aligned}
& \int_{0}^{\infty} g(y)\left[\int_{0}^{\frac{1}{y}} \frac{(\min \{x y, 1\})^{\alpha}|\ln x y|^{\beta}}{(\max \{x y, 1\})^{\lambda+\alpha}} f(x) d x\right] d y \\
& \quad \leq M_{1}\left[\int_{0}^{\infty} x^{p(1-\sigma)-1} f^{p}(x) d x\right]^{\frac{1}{p}}\left[\int_{0}^{\infty} y^{q\left(1-\sigma_{1}\right)-1} g^{q}(y) d y\right]^{\frac{1}{q}}
\end{aligned}
$$

holds true, then we have $\sigma_{1}=\sigma$, and then $M_{1} \geq k_{1}(\sigma)$.

Proof If $\sigma_{1}>\sigma$, then, for $n \geq \frac{1}{\sigma_{1}-\sigma}(n \in \mathbf{N})$, we set the following functions:

$$
f_{n}(x):=\left\{\begin{array}{ll}
x^{\sigma+\frac{1}{p n}-1}, & 0<x \leq 1, \\
0, & x>1,
\end{array} \quad g_{n}(y):= \begin{cases}0, & 0<y<1 \\
y^{\sigma_{1}-\frac{1}{q^{n}}-1}, & y \geq 1\end{cases}\right.
$$

and find

$$
\begin{aligned}
J_{1} & :=\left[\int_{0}^{\infty} x^{p(1-\sigma)-1} f_{n}^{p}(x) d x\right]^{\frac{1}{p}}\left[\int_{0}^{\infty} y^{q\left(1-\sigma_{1}\right)-1} g_{n}^{q}(y) d y\right]^{\frac{1}{q}} \\
& =\left(\int_{0}^{1} x^{\frac{1}{n}-1} d x\right)^{\frac{1}{p}}\left(\int_{1}^{\infty} y^{-\frac{1}{n}-1} d y\right)^{\frac{1}{q}}=n .
\end{aligned}
$$

Setting $u=x y$, we obtain

$$
\begin{aligned}
I_{1} & :=\int_{0}^{\infty} g_{n}(y)\left(\int_{0}^{\frac{1}{y}} \frac{(\min \{x y, 1\})^{\alpha}|\ln x y|^{\beta}}{(\max \{x y, 1\})^{\lambda+\alpha}} f_{n}(x) d x\right) d y \\
& =\int_{1}^{\infty}\left(\int_{0}^{\frac{1}{y}} \frac{(\min \{x y, 1\})^{\alpha}(-\ln x y)^{\beta}}{(\max \{x y, 1\})^{\lambda+\alpha}} x^{\sigma+\frac{1}{p n}-1} d x\right) y^{\sigma_{1}-\frac{1}{q n}-1} d y \\
& =\int_{1}^{\infty} y^{\left(\sigma_{1}-\sigma\right)-\frac{1}{n}-1} d y \int_{0}^{1} \frac{(\min \{u, 1\})^{\alpha}(-\ln u)^{\beta}}{(\max \{u, 1\})^{\lambda+\alpha}} u^{\sigma+\frac{1}{p n}-1} d u .
\end{aligned}
$$

Then by (13), we have

$$
\begin{aligned}
& \int_{1}^{\infty} y^{\left(\sigma_{1}-\sigma\right)-\frac{1}{n}-1} d y \int_{0}^{1} \frac{(\min \{u, 1\})^{\alpha}(-\ln u)^{\beta}}{(\max \{u, 1\})^{\lambda+\alpha}} u^{\sigma+\frac{1}{p n}-1} d u \\
& \quad=I_{1} \leq M_{1} J_{1}=M_{1} n<\infty .
\end{aligned}
$$


Since $\left(\sigma_{1}-\sigma\right)-\frac{1}{n} \geq 0$, it follows that $\int_{1}^{\infty} y^{\left(\sigma_{1}-\sigma\right)-\frac{1}{n}-1} d y=\infty$. By (14), in view of $\int_{0}^{\frac{1}{y}} \frac{(\min \{u, 1\})^{\alpha}(-\ln u)^{\beta}}{(\max \{u, 1\})^{\lambda+\alpha}} u^{\sigma+\frac{1}{p n}-1} d u>0$, we find $\infty<\infty$, which is a contradiction.

If $\sigma_{1}<\sigma$, then, for $n \geq \frac{1}{\sigma-\sigma_{1}}(n \in \mathbf{N})$, we set the following functions:

$$
\tilde{f}_{n}(x):=\left\{\begin{array}{ll}
0, & 0<x<1, \\
x^{\sigma-\frac{1}{p n}-1}, & x \geq 1,
\end{array} \quad \tilde{g}_{n}(y):= \begin{cases}y^{\sigma_{1}+\frac{1}{q^{n}}-1}, & 0<y \leq 1 \\
0, & y>1,\end{cases}\right.
$$

and find

$$
\begin{aligned}
\tilde{J}_{1} & :=\left[\int_{0}^{\infty} x^{p(1-\sigma)-1} \tilde{f}_{n}^{p}(x) d x\right]^{\frac{1}{p}}\left[\int_{0}^{\infty} y^{q\left(1-\sigma_{1}\right)-1} \tilde{g}_{n}^{q}(y) d y\right]^{\frac{1}{q}} \\
& =\left(\int_{1}^{\infty} x^{-\frac{1}{n}-1} d x\right)^{\frac{1}{p}}\left(\int_{0}^{1} y^{\frac{1}{n}-1} d y\right)^{\frac{1}{q}}=n .
\end{aligned}
$$

Setting $u=x y$, we obtain

$$
\begin{aligned}
\tilde{I}_{1} & :=\int_{0}^{\infty} \tilde{f}_{n}(x)\left(\int_{0}^{\frac{1}{x}} \frac{(\min \{x y, 1\})^{\alpha}|\ln x y|^{\beta}}{(\max \{x y, 1\})^{\lambda+\alpha}} \tilde{g}_{n}(y) d y\right) d x \\
& =\int_{1}^{\infty}\left(\int_{0}^{\frac{1}{x}} \frac{(\min \{x y, 1\})^{\alpha}(-\ln x y)^{\beta}}{(\max \{x y, 1\})^{\lambda+\alpha}} y^{\sigma_{1}+\frac{1}{q^{n}}-1} d y\right) x^{\sigma-\frac{1}{p^{n}}-1} d x \\
& =\int_{1}^{\infty} x^{\left(\sigma-\sigma_{1}\right)-\frac{1}{n}-1} d x \int_{0}^{1} \frac{(\min \{u, 1\})^{\alpha}(-\ln u)^{\beta}}{(\max \{u, 1\})^{\lambda+\alpha}} u^{\sigma_{1}+\frac{1}{q^{n}}-1} d u .
\end{aligned}
$$

Then by the Fubini theorem $(c f .[20])$ and (13), we have

$$
\begin{aligned}
& \int_{1}^{\infty} x^{\left(\sigma-\sigma_{1}\right)-\frac{1}{n}-1} d x \int_{0}^{1} \frac{(\min \{u, 1\})^{\alpha}(-\ln u)^{\beta}}{(\max \{u, 1\})^{\lambda+\alpha}} u^{\sigma_{1}+\frac{1}{q n}-1} d u \\
& =\tilde{I}_{1}=\int_{0}^{\infty} \tilde{g}_{n}(y)\left(\int_{0}^{\frac{1}{y}} \frac{(\min \{x y, 1\})^{\alpha}|\ln x y|^{\beta}}{(\max \{x y, 1\})^{\lambda+\alpha}} \tilde{f}_{n}(x) d x\right) d y \\
& \leq M_{1} \tilde{J}_{1}=M_{1} n<\infty
\end{aligned}
$$

Since $\left(\sigma_{1}-\sigma\right)-\frac{1}{n} \geq 0$, it follows that $\int_{1}^{\infty} x^{\left(\sigma-\sigma_{1}\right)-\frac{1}{n}-1} d x=\infty$. By (15), in view of

$$
\int_{0}^{1} \frac{(\min \{u, 1\})^{\alpha}(-\ln u)^{\beta}}{(\max \{u, 1\})^{\lambda+\alpha}} u^{\sigma_{1}+\frac{1}{q^{n}}-1} d u>0
$$

we find $\infty<\infty$, which is a contradiction.

Hence, we conclude that $\sigma_{1}=\sigma$.

For $\sigma_{1}=\sigma$, we reduce (15) as follows:

$$
M_{1} \geq \int_{0}^{1} \frac{(\min \{u, 1\})^{\alpha}(-\ln u)^{\beta}}{(\max \{u, 1\})^{\lambda+\alpha}} u^{\sigma+\frac{1}{q^{n}}-1} d u
$$

Since

$$
\left\{\frac{(\min \{u, 1\})^{\alpha}(-\ln u)^{\beta}}{(\max \{u, 1\})^{\lambda+\alpha}} u^{\sigma+\frac{1}{q^{n}}-1}\right\}_{n=1}^{\infty}
$$


is non-negative and increasing in $(0,1]$, by Levi theorem $(c f .[20])$, we find

$$
\begin{aligned}
M_{1} & \geq \lim _{n \rightarrow \infty} \int_{0}^{1} \frac{(\min \{u, 1\})^{\alpha}(-\ln u)^{\beta}}{(\max \{u, 1\})^{\lambda+\alpha}} u^{\sigma+\frac{1}{q^{n}}-1} d u \\
& =\int_{0}^{1} \lim _{n \rightarrow \infty} \frac{(\min \{u, 1\})^{\alpha}(-\ln u)^{\beta}}{(\max \{u, 1\})^{\lambda+\alpha}} u^{\sigma+\frac{1}{q^{n}}-1} d u=k_{1}(\sigma) .
\end{aligned}
$$

The lemma is proved.

Lemma 2 If $p>1, \frac{1}{p}+\frac{1}{q}=1, \sigma_{1} \in R, \beta>-1, \mu=\lambda-\sigma>-\alpha$, there exists a constant $M_{2}$, such that, for any non-negative measurable functions $f(x)$ and $g(y)$ in $(0, \infty)$, the following inequality:

$$
\begin{aligned}
& \int_{0}^{\infty} g(y)\left[\int_{\frac{1}{y}}^{\infty} \frac{(\min \{x y, 1\})^{\alpha}|\ln x y|^{\beta}}{(\max \{x y, 1\})^{\lambda+\alpha}} f(x) d x\right] d y \\
& \quad \leq M_{2}\left[\int_{0}^{\infty} x^{p(1-\sigma)-1} f^{p}(x) d x\right]^{\frac{1}{p}}\left[\int_{0}^{\infty} y^{q\left(1-\sigma_{1}\right)-1} g^{q}(y) d y\right]^{\frac{1}{q}}
\end{aligned}
$$

holds true, then we have $\sigma_{1}=\sigma$, and then $M_{2} \geq k_{1}(\mu)$.

Proof If $\sigma_{1}<\sigma$, then, for $n \geq \frac{1}{\sigma-\sigma_{1}}(n \in \mathbf{N})$, we set two functions $\tilde{f}_{n}(x)$ and $\tilde{g}_{n}(y)$ as in Lemma 1 , and find

$$
\tilde{J}_{1}=\left[\int_{0}^{\infty} x^{p(1-\sigma)-1} \tilde{f}_{n}^{p}(x) d x\right]^{\frac{1}{p}}\left[\int_{0}^{\infty} y^{q\left(1-\sigma_{1}\right)-1} \tilde{g}_{n}^{q}(y) d y\right]^{\frac{1}{q}}=n .
$$

Setting $u=x y$, we obtain

$$
\begin{aligned}
\tilde{I}_{2} & :=\int_{0}^{\infty} \tilde{g}_{n}(y)\left(\int_{\frac{1}{y}}^{\infty} \frac{(\min \{x y, 1\})^{\alpha}|\ln x y|^{\beta}}{(\max \{x y, 1\})^{\lambda+\alpha}} \tilde{f}_{n}(x) d x\right) d y \\
& =\int_{0}^{1}\left(\int_{\frac{1}{y}}^{\infty} \frac{(\min \{x y, 1\})^{\alpha}(\ln x y)^{\beta}}{(\max \{x y, 1\})^{\lambda+\alpha}} x^{\sigma-\frac{1}{p n}-1} d x\right) y^{\sigma_{1}+\frac{1}{q n}-1} d y \\
& =\int_{0}^{1} y^{\left(\sigma_{1}-\sigma\right)+\frac{1}{n}-1} d y \int_{1}^{\infty} \frac{(\min \{u, 1\})^{\alpha}(\ln u)^{\beta}}{(\max \{u, 1\})^{\lambda+\alpha}} u^{\sigma-\frac{1}{p n}-1} d u,
\end{aligned}
$$

and then by (17), we obtain

$$
\begin{aligned}
& \int_{0}^{1} y^{\left(\sigma_{1}-\sigma\right)+\frac{1}{n}-1} d y \int_{1}^{\infty} \frac{(\min \{u, 1\})^{\alpha}(\ln u)^{\beta}}{(\max \{u, 1\})^{\lambda+\alpha}} u^{\sigma-\frac{1}{p n}-1} d u \\
& \quad=\tilde{I}_{2} \leq M_{2} \tilde{J}_{1}=M_{2} n<\infty .
\end{aligned}
$$

Since $\left(\sigma_{1}-\sigma\right)+\frac{1}{n} \leq 0$, it follows that $\int_{1}^{\infty} y^{\left(\sigma_{1}-\sigma\right)+\frac{1}{n}-1} d y=\infty$. By (18), in view of

$$
\int_{1}^{\infty} \frac{(\min \{u, 1\})^{\alpha}(\ln u)^{\beta}}{(\max \{u, 1\})^{\lambda+\alpha}} u^{\sigma-\frac{1}{p n}-1} d u>0,
$$

we have $\infty<\infty$, which is a contradiction. 
If $\sigma_{1}>\sigma$, then, for $n \geq \frac{1}{\sigma_{1}-\sigma}(n \in \mathbf{N})$, we set two functions $f_{n}(x)$ and $g_{n}(y)$ as in Lemma 1 , and we find

$$
J_{1}=\left[\int_{0}^{\infty} x^{p(1-\sigma)-1} f_{n}^{p}(x) d x\right]^{\frac{1}{p}}\left[\int_{0}^{\infty} y^{q\left(1-\sigma_{1}\right)-1} g_{n}^{q}(y) d y\right]^{\frac{1}{q}}=n
$$

Setting $u=x y$, we obtain

$$
\begin{aligned}
I_{2} & :=\int_{0}^{\infty} f_{n}(x)\left(\int_{\frac{1}{x}}^{\infty} \frac{(\min \{x y, 1\})^{\alpha}|\ln x y|^{\beta}}{(\max \{x y, 1\})^{\lambda+\alpha}} g_{n}(y) d y\right) d x \\
& =\int_{0}^{1}\left[\int_{\frac{1}{x}}^{\infty} \frac{(\min \{x y, 1\})^{\alpha}(\ln x y)^{\beta}}{(\max \{x y, 1\})^{\lambda+\alpha}} y^{\sigma_{1}-\frac{1}{q^{n}}-1} d y\right] x^{\sigma+\frac{1}{p^{n}}-1} d x \\
& =\int_{0}^{1} x^{\left(\sigma-\sigma_{1}\right)+\frac{1}{n}-1} d x \int_{1}^{\infty} \frac{(\min \{u, 1\})^{\alpha}(\ln u)^{\beta}}{(\max \{u, 1\})^{\lambda+\alpha}} u^{\sigma_{1}-\frac{1}{q n}-1} d u
\end{aligned}
$$

and then by Fubini theorem (cf. [20]) and (17), we have

$$
\begin{aligned}
& \int_{0}^{1} x^{\left(\sigma-\sigma_{1}\right)+\frac{1}{n}-1} d x \int_{1}^{\infty} \frac{(\min \{u, 1\})^{\alpha}(\ln u)^{\beta}}{(\max \{u, 1\})^{\lambda+\alpha}} u^{\sigma_{1}-\frac{1}{q n}-1} d u \\
& \quad=I_{2}=\int_{0}^{\infty} g_{n}(y)\left(\int_{\frac{1}{y}}^{\infty} \frac{(\min \{x y, 1\})^{\alpha}(\ln x y)^{\beta}}{(\max \{x y, 1\})^{\lambda+\alpha}} f_{n}(x) d x\right) d y \\
& \leq M_{2} J_{1}=M_{2} n
\end{aligned}
$$

Since $\left(\sigma-\sigma_{1}\right)+\frac{1}{n} \leq 0$, it follows that $\int_{0}^{1} x^{\left(\sigma-\sigma_{1}\right)+\frac{1}{n}-1} d x=\infty$. By (19), in view of

$$
\int_{1}^{\infty} \frac{(\min \{u, 1\})^{\alpha}(\ln u)^{\beta}}{(\max \{u, 1\})^{\lambda+\alpha}} u^{\sigma_{1-\frac{1}{q n}}-1} d u>0
$$

we have $\infty<\infty$, which is a contradiction.

Hence, we conclude that $\sigma_{1}=\sigma$.

For $\sigma_{1}=\sigma$, we reduce (19) as follows:

$$
M_{2} \geq \int_{1}^{\infty} \frac{(\min \{u, 1\})^{\alpha}(\ln u)^{\beta}}{(\max \{u, 1\})^{\lambda+\alpha}} u^{\sigma-\frac{1}{q^{n}}-1} d u
$$

Since

$$
\left\{\frac{(\min \{u, 1\})^{\alpha}(\ln u)^{\beta}}{(\max \{u, 1\})^{\lambda+\alpha}} u^{\sigma-\frac{1}{q n}-1}\right\}_{n=1}^{\infty}
$$

is non-negative and increasing in $[1, \infty)$, still by the Levi theorem $(c f .[20])$, we have

$$
\begin{aligned}
M_{2} & \geq \lim _{n \rightarrow \infty} \int_{1}^{\infty} \frac{(\min \{u, 1\})^{\alpha}(\ln u)^{\beta}}{(\max \{u, 1\})^{\lambda+\alpha}} u^{\sigma-\frac{1}{q^{n}}-1} d u \\
& =\int_{1}^{\infty} \lim _{n \rightarrow \infty} \frac{(\min \{u, 1\})^{\alpha}(\ln u)^{\beta}}{(\max \{u, 1\})^{\lambda+\alpha}} u^{\sigma-\frac{1}{q^{n}}-1} d u=k_{1}(\mu) .
\end{aligned}
$$

The lemma is proved. 


\section{Main results and corollaries}

Theorem 1 If $p>1, \frac{1}{p}+\frac{1}{q}=1, \sigma_{1} \in R, \beta>-1, \sigma>-\alpha$, then the following conditions are equivalent:

(i) There exists a constant $M_{1}$, such that, for any $f(x) \geq 0$, satisfying

$$
0<\int_{0}^{\infty} x^{p(1-\sigma)-1} f^{p}(x) d x<\infty
$$

we have the following Hardy-type integral inequality of the first kind with the non-homogeneous kernel:

$$
\begin{aligned}
J & :=\left\{\int_{0}^{\infty} y^{p \sigma_{1}-1}\left[\int_{0}^{\frac{1}{y}} \frac{(\min \{x y, 1\})^{\alpha}|\ln x y|^{\beta}}{(\max \{x y, 1\})^{\lambda+\alpha}} f(x) d x\right]^{p} d y\right\}^{\frac{1}{p}} \\
& <M_{1}\left[\int_{0}^{\infty} x^{p(1-\sigma)-1} f^{p}(x) d x\right]^{\frac{1}{p}} .
\end{aligned}
$$

(ii) There exists a constant $M_{1}$, such that, for any $f(x), g(y) \geq 0$, satisfying $0<\int_{0}^{\infty} x^{p(1-\sigma)-1} f^{p}(x) d x<\infty$, and $0<\int_{0}^{\infty} y^{q\left(1-\sigma_{1}\right)-1} g^{q}(y) d y<\infty$, we have the following inequality:

$$
\begin{aligned}
I & :=\int_{0}^{\infty} g(y)\left[\int_{0}^{\frac{1}{y}} \frac{(\min \{x y, 1\})^{\alpha}|\ln x y|^{\beta}}{(\max \{x y, 1\})^{\lambda+\alpha}} f(x) d x\right] d y \\
& <M_{1}\left[\int_{0}^{\infty} x^{p(1-\sigma)-1} f^{p}(x) d x\right]^{\frac{1}{p}}\left[\int_{0}^{\infty} y^{q\left(1-\sigma_{1}\right)-1} g^{q}(y) d y\right]^{\frac{1}{q}} .
\end{aligned}
$$

(iii) $\sigma_{1}=\sigma$.

If condition (iii) holds true, then $M_{1} \geq k_{1}(\sigma)$ and the constant factor

$$
M_{1}=k_{1}(\sigma)=\frac{\Gamma(\beta+1)}{(\sigma+\alpha)^{\beta+1}}
$$

in (21) and (22) is the best possible.

Proof (i) $\Rightarrow$ (ii). By Hölder's inequality (cf. [21]), we have

$$
\begin{aligned}
I & =\int_{0}^{\infty}\left[y^{\sigma_{1}-\frac{1}{p}} \int_{0}^{\frac{1}{y}} \frac{(\min \{x y, 1\})^{\alpha}|\ln x y|^{\beta}}{(\max \{x y, 1\})^{\lambda+\alpha}} f(x) d x\right]\left(y^{\frac{1}{p}-\sigma_{1}} g(y)\right) d y \\
& \leq J\left[\int_{0}^{\infty} y^{q\left(1-\sigma_{1}\right)-1} g^{q}(y) d y\right]^{\frac{1}{q}} .
\end{aligned}
$$

Then by (21), we have (22).

(ii) $\Rightarrow$ (iii). By Lemma 1, we have $\sigma_{1}=\sigma$.

(iii) $\Rightarrow($ i). Setting $u=x y$, we obtain the following weight function:

$$
\begin{aligned}
\omega_{1}(\sigma, y) & :=y^{\sigma} \int_{0}^{\frac{1}{y}} \frac{(\min \{x y, 1\})^{\alpha}|\ln x y|^{\beta}}{(\max \{x y, 1\})^{\lambda+\alpha}} x^{\sigma-1} d x \\
& =\int_{0}^{1} \frac{(\min \{u, 1\})^{\alpha}(-\ln u)^{\beta}}{(\max \{u, 1\})^{\lambda+\alpha}} u^{\sigma-1} d u=k_{1}(\sigma) \quad(y>0) .
\end{aligned}
$$


By Hölder's inequality with weight and (24), for $y \in(0, \infty)$, we have

$$
\begin{aligned}
& {\left[\int_{0}^{\frac{1}{y}} \frac{(\min \{x y, 1\})^{\alpha}|\ln x y|^{\beta}}{(\max \{x y, 1\})^{\lambda+\alpha}} f(x) d x\right]^{p} } \\
&=\left\{\int_{0}^{\frac{1}{y}} \frac{(\min \{x y, 1\})^{\alpha}|\ln x y|^{\beta}}{(\max \{x y, 1\})^{\lambda+\alpha}}\left[\frac{y^{(\sigma-1) / p}}{x^{(\sigma-1) / q}} f(x)\right]\left[\frac{x^{(\sigma-1) / q}}{y^{(\sigma-1) / p}}\right] d x\right\}^{p} \\
& \leq \int_{0}^{\frac{1}{y}} \frac{(\min \{x y, 1\})^{\alpha}|\ln x y|^{\beta}}{(\max \{x y, 1\})^{\lambda+\alpha}} \frac{y^{\sigma-1} f^{p}(x)}{x^{(\sigma-1) p / q}} d x \\
& \times\left[\int_{0}^{\frac{1}{y}} \frac{(\min \{x y, 1\})^{\alpha}|\ln x y|^{\beta}}{(\max \{x y, 1\})^{\lambda+\alpha}} \frac{x^{\sigma-1}}{y^{(\sigma-1) q / p}} d x\right]^{p-1} \\
&= {\left[\frac{\omega_{1}(\sigma, y)}{y^{q(\sigma-1)+1}}\right]_{0}^{p-1} \int_{0}^{\frac{1}{y}} \frac{(\min \{x y, 1\})^{\alpha}|\ln x y|^{\beta}}{(\max \{x y, 1\})^{\lambda+\alpha}} \frac{y^{\sigma-1}}{x^{(\sigma-1) p / q}} f^{p}(x) d x } \\
&=\left(k_{1}(\sigma)\right)^{p-1} y^{-p \sigma+1} \int_{0}^{\frac{1}{y}} \frac{(\min \{x y, 1\})^{\alpha}|\ln x y|^{\beta}}{(\max \{x y, 1\})^{\lambda+\alpha}} \frac{y^{\sigma-1}}{x^{(\sigma-1) p / q}} f^{p}(x) d x .
\end{aligned}
$$

If (25) obtains the form of equality for a $y \in(0, \infty)$, then $(c f$. [21]), there exist constants $A$ and $B$, such that they are not all zero, and

$$
A \frac{y^{\sigma-1}}{x^{(\sigma-1) p / q}} f^{p}(x)=B \frac{x^{\sigma-1}}{y^{(\sigma-1) q / p}} \quad \text { a.e. in } \mathbf{R}_{+}
$$

We suppose that $A \neq 0$ (otherwise $B=A=0$ ). It follows that

$$
x^{p(1-\sigma)-1} f^{p}(x)=y^{q(\sigma-1)} \frac{B}{A x} \quad \text { a.e. in } \mathbf{R}_{+},
$$

which contradicts the fact that $0<\int_{0}^{\infty} x^{p(1-\sigma)-1} f^{p}(x) d x<\infty$. Hence, (25) assumes the form of strict inequality. Hence, for $\sigma_{1}=\sigma$, by (25) and by the Fubini theorem (cf. [20]), we obtain

$$
\begin{aligned}
J & <\left(k_{1}(\sigma)\right)^{\frac{1}{q}}\left\{\int_{0}^{\infty}\left[\int_{0}^{\frac{1}{y}} \frac{(\min \{x y, 1\})^{\alpha}|\ln x y|^{\beta}}{(\max \{x y, 1\})^{\lambda+\alpha}} \frac{y^{\sigma-1} f^{p}(x)}{x^{(\sigma-1) p / q}} d x\right] d y\right\}^{\frac{1}{p}} \\
& =\left(k_{1}(\sigma)\right)^{\frac{1}{q}}\left\{\int_{0}^{\infty}\left[\int_{0}^{\frac{1}{x}} \frac{(\min \{x y, 1\})^{\alpha}|\ln x y|^{\beta}}{(\max \{x y, 1\})^{\lambda+\alpha}} \frac{y^{\sigma-1} d y}{x^{(\sigma-1)(p-1)}}\right] f^{p}(x) d x\right\}^{\frac{1}{p}} \\
& =\left(k_{1}(\sigma)\right)^{\frac{1}{q}}\left[\int_{0}^{\infty} \omega_{1}(\sigma, x) x^{p(1-\sigma)-1} f^{p}(x) d x\right]^{\frac{1}{p}} \\
& =k_{1}(\sigma)\left[\int_{0}^{\infty} x^{p(1-\sigma)-1} f^{p}(x) d x\right]^{\frac{1}{p}} .
\end{aligned}
$$

Setting $M_{1} \geq k_{1}(\sigma)$, (21) follows.

Therefore, conditions (i), (ii) and (iii) are equivalent.

When condition (iii) follows, if there exists a constant factor $M_{1} \geq k_{1}(\sigma)$, such that (22) is valid, then by Lemma 1 , we have $M_{1} \geq k_{1}(\sigma)$. Hence, the constant factor $M_{1}=k_{1}(\sigma)$ in (22) is the best possible. The constant factor $M_{1}=k_{1}(\sigma)$ in (21) is still the best possible. Otherwise, by (23) (for $\sigma_{1}=\sigma$ ), we can conclude that the constant factor $M_{1}=k_{1}(\sigma)$ in (22) is not the best possible. 
Setting $y=\frac{1}{Y}, G(Y)=Y^{\lambda-2} g\left(\frac{1}{Y}\right), \mu_{1}=\lambda-\sigma_{1}$ in Theorem 1 , then replacing $Y$ by $y$ and $G(Y)$ by $g(y)$, we have

Corollary 1 If $p>1, \frac{1}{p}+\frac{1}{q}=1, \mu_{1} \in R, \beta>-1, \sigma=\lambda-\mu>-\alpha$, then the following conditions are equivalent:

(i) There exists a constant $M_{1}$, such that, for any $f(x) \geq 0$, satisfying

$$
0<\int_{0}^{\infty} x^{p(1-\sigma)-1} f^{p}(x) d x<\infty
$$

we have the following Hardy-type inequality of the first kind with the homogeneous kernel:

$$
\begin{aligned}
& \left\{\int_{0}^{\infty} y^{p \mu_{1}-1}\left[\int_{0}^{y} \frac{(\min \{x, y\})^{\alpha}|\ln (x / y)|^{\beta}}{(\max \{x, y\})^{\lambda+\alpha}} f(x) d x\right]^{p} d y\right\}^{\frac{1}{p}} \\
& \quad<M_{1}\left[\int_{0}^{\infty} x^{p(1-\sigma)-1} f^{p}(x) d x\right]^{\frac{1}{p}} .
\end{aligned}
$$

(ii) There exists a constant $M_{1}$, such that, for any $f(x), g(y) \geq 0$, satisfying $0<\int_{0}^{\infty} x^{p(1-\sigma)-1} f^{p}(x) d x<\infty$, and $0<\int_{0}^{\infty} y^{q\left(1-\mu_{1}\right)-1} g^{q}(y) d y<\infty$, we have the following inequality:

$$
\begin{aligned}
& \int_{0}^{\infty} g(y)\left[\int_{0}^{y} \frac{(\min \{x, y\})^{\alpha}|\ln (x / y)|^{\beta}}{(\max \{x, y\})^{\lambda+\alpha}} f(x) d x\right] d y \\
& \quad<M_{1}\left[\int_{0}^{\infty} x^{p(1-\sigma)-1} f^{p}(x) d x\right]^{\frac{1}{p}}\left[\int_{0}^{\infty} y^{q\left(1-\mu_{1}\right)-1} g^{q}(y) d y\right]^{\frac{1}{q}} .
\end{aligned}
$$

(iii) $\mu_{1}=\mu$.

If condition (iii) holds true, then we have $M_{1} \geq k_{1}(\sigma)$, and the constant $M_{1}=k_{1}(\sigma)$ in (26) and (27) is the best possible.

Remark 2 On the other hand, setting $y=\frac{1}{Y}, G(Y)=Y^{\lambda-2} g\left(\frac{1}{Y}\right), \sigma_{1}=\lambda-\mu_{1}$ in Corollary 1 , then replacing $Y$ by $y$ and $G(Y)$ by $g(y)$, we have Theorem 1 . Hence, Theorem 1 and Corollary 1 are equivalent.

Similarly, we obtain the following weight function:

$$
\begin{aligned}
\omega_{2}(\sigma, y) & :=y^{\sigma} \int_{\frac{1}{y}}^{\infty} \frac{(\min \{x y, 1\})^{\alpha}|\ln x y|^{\beta}}{(\max \{x y, 1\})^{\lambda+\alpha}} x^{\sigma-1} d x \\
& =\int_{1}^{\infty} \frac{(\min \{u, 1\})^{\alpha}|\ln u|^{\beta}}{(\max \{u, 1\})^{\lambda+\alpha}} u^{\sigma-1} d u=k_{1}(\mu) \quad(y>0),
\end{aligned}
$$

and then in view of Lemma 2 and in the same way, we have

Theorem 2 If $p>1, \frac{1}{p}+\frac{1}{q}=1, \sigma_{1} \in R, \beta>-1, \mu=\lambda-\sigma>-\alpha$, then the following conditions are equivalent: 
(i) There exists a constant $M_{2}$, such that, for any $f(x) \geq 0$, satisfying

$$
0<\int_{0}^{\infty} x^{p(1-\sigma)-1} f^{p}(x) d x<\infty
$$

we have the following Hardy-type inequality of the second kind with the non-homogeneous kernel:

$$
\begin{aligned}
& \left\{\int_{0}^{\infty} y^{p \sigma_{1}-1}\left[\int_{\frac{1}{y}}^{\infty} \frac{(\min \{x y, 1\})^{\alpha}|\ln x y|^{\beta}}{(\max \{x y, 1\})^{\lambda+\alpha}} f(x) d x\right]^{p} d y\right\}^{\frac{1}{p}} \\
& \quad<M_{2}\left[\int_{0}^{\infty} x^{p(1-\sigma)-1} f^{p}(x) d x\right]^{\frac{1}{p}}
\end{aligned}
$$

(ii) There exists a constant $M_{2}$, such that, for any $f(x), g(y) \geq 0$, satisfying $0<\int_{0}^{\infty} x^{p(1-\sigma)-1} f^{p}(x) d x<\infty$, and $0<\int_{0}^{\infty} y^{q\left(1-\sigma_{1}\right)-1} g^{q}(y) d y<\infty$, we have the following inequality:

$$
\begin{aligned}
& \int_{0}^{\infty} g(y)\left[\int_{\frac{1}{y}}^{\infty} \frac{(\min \{x y, 1\})^{\alpha}|\ln x y|^{\beta}}{(\max \{x y, 1\})^{\lambda+\alpha}} f(x) d x\right] d y \\
& \quad<M_{2}\left[\int_{0}^{\infty} x^{p(1-\sigma)-1} f^{p}(x) d x\right]^{\frac{1}{p}}\left[\int_{0}^{\infty} y^{q\left(1-\sigma_{1}\right)-1} g^{q}(y) d y\right]^{\frac{1}{q}} .
\end{aligned}
$$

(iii) $\sigma_{1}=\sigma$.

If condition (iii) holds true, then we have $M_{2} \geq k_{1}(\mu)$ and the constant factor

$$
M_{2}=k_{1}(\mu)=\frac{\Gamma(\beta+1)}{(\mu+\alpha)^{\beta+1}}
$$

in (28) and (29) is the best possible.

Setting $y=\frac{1}{Y}, G(Y)=Y^{\lambda-2} g\left(\frac{1}{Y}\right), \mu_{1}=\lambda-\sigma_{1}$ in Theorem 2, then replacing $Y$ by $y$ and $G(Y)$ by $g(y)$, we have

Corollary 2 If $p>1, \frac{1}{p}+\frac{1}{q}=1, \mu_{1} \in R, \beta>-1, \mu=\lambda-\sigma>-\alpha$, then the following conditions are equivalent:

(i) There exists a constant $M_{2}$, such that, for any $f(x) \geq 0$, satisfying

$$
0<\int_{0}^{\infty} x^{p(1-\sigma)-1} f^{p}(x) d x<\infty
$$

we have the following Hardy-type inequality of the second kind with the homogeneous kernel:

$$
\begin{aligned}
& \left\{\int_{0}^{\infty} y^{p \mu_{1}-1}\left[\int_{y}^{\infty} \frac{(\min \{x, y\})^{\alpha}|\ln (x / y)|^{\beta}}{(\max \{x, y\})^{\lambda+\alpha}} f(x) d x\right]^{p} d y\right\}^{\frac{1}{p}} \\
& \quad<M_{2}\left[\int_{0}^{\infty} x^{p(1-\sigma)-1} f^{p}(x) d x\right]^{\frac{1}{p}}
\end{aligned}
$$


(ii) There exists a constant $M_{2}$, such that, for any $f(x), g(y) \geq 0$, satisfying $0<\int_{0}^{\infty} x^{p(1-\sigma)-1} f^{p}(x) d x<\infty$, and $0<\int_{0}^{\infty} y^{q\left(1-\mu_{1}\right)-1} g^{q}(y) d y<\infty$, we have the following inequality:

$$
\begin{aligned}
& \int_{0}^{\infty} g(y)\left[\int_{y}^{\infty} \frac{(\min \{x, y\})^{\alpha}|\ln (x / y)|^{\beta}}{(\max \{x, y\})^{\lambda+\alpha}} f(x) d x\right] d y \\
& \quad<M_{2}\left[\int_{0}^{\infty} x^{p(1-\sigma)-1} f^{p}(x) d x\right]^{\frac{1}{p}}\left[\int_{0}^{\infty} y^{q\left(1-\mu_{1}\right)-1} g^{q}(y) d y\right]^{\frac{1}{q}} .
\end{aligned}
$$

(iii) $\mu_{1}=\mu$.

If condition (iii) holds true, then we have $M_{2} \geq k_{1}(\mu)$, and the constant $M_{2}=k_{1}(\mu)$ in (30) and (31) is the best possible.

Remark 3 Theorem 2 and Corollary 2 are still equivalent.

\section{Operator expressions}

For $p>1, \frac{1}{p}+\frac{1}{q}=1, \sigma, \lambda>0, \mu=\lambda-\sigma$, we set the following functions: $\varphi(x):=x^{p(1-\sigma)-1}$, $\psi(y):=y^{q(1-\sigma)-1}, \phi(y):=y^{q(1-\mu)-1}$, wherefrom, $\psi^{1-p}(y)=y^{p \sigma-1}, \phi^{1-p}(y)=y^{p \mu-1}\left(x, y \in \mathbf{R}_{+}\right)$.

Define the following real normed linear spaces:

$$
L_{p, \varphi}\left(\mathbf{R}_{+}\right):=\left\{f:\|f\|_{p, \varphi}:=\left(\int_{0}^{\infty} \varphi(x)|f(x)|^{p} d x\right)^{\frac{1}{p}}<\infty\right\}
$$

wherefrom

$$
\begin{aligned}
& L_{q, \psi}\left(\mathbf{R}_{+}\right):=\left\{g:\|g\|_{q, \psi}:=\left(\int_{0}^{\infty} \psi(y)|g(y)|^{q} d y\right)^{\frac{1}{q}}<\infty\right\}, \\
& L_{q, \phi}\left(\mathbf{R}_{+}\right):=\left\{g:\|g\|_{q, \phi}:=\left(\int_{0}^{\infty} \phi(y)|g(y)|^{q} d y\right)^{\frac{1}{q}}<\infty\right\}, \\
& L_{p, \psi^{1}-p}\left(\mathbf{R}_{+}\right)=\left\{h:\|h\|_{p, \psi^{1-p}}=\left(\int_{0}^{\infty} \psi^{1-p}(y)|h(y)|^{p} d y\right)^{\frac{1}{p}}<\infty\right\}, \\
& L_{q, \phi^{1-p}}\left(\mathbf{R}_{+}\right)=\left\{h:\|h\|_{p, \phi^{1-p}}=\left(\int_{0}^{\infty} \phi^{1-p}(y)|h(y)|^{p} d y\right)^{\frac{1}{p}}<\infty\right\} .
\end{aligned}
$$

(a) In view of Theorem $1\left(\sigma_{1}=\sigma\right)$, for $f \in L_{p, \varphi}\left(\mathbf{R}_{+}\right)$, setting

$$
h_{1}(y):=\int_{0}^{\frac{1}{y}} \frac{(\min \{x y, 1\})^{\alpha}|\ln x y|^{\beta}}{(\max \{x y, 1\})^{\lambda+\alpha}} f(x) d x \quad\left(y \in \mathbf{R}_{+}\right)
$$

by (21), we have

$$
\left\|h_{1}\right\|_{p, \psi^{1-p}}=\left[\int_{0}^{\infty} \psi^{1-p}(y) h_{1}^{p}(y) d y\right]^{\frac{1}{p}}<M_{1}\|f\|_{p, \varphi}<\infty .
$$

Definition 1 Define a Hardy-type integral operator of the first kind with the nonhomogeneous kernel $T_{1}^{(1)}: L_{p, \varphi}\left(\mathbf{R}_{+}\right) \rightarrow L_{p, \psi^{1-p}}\left(\mathbf{R}_{+}\right)$as follows: For any $f \in L_{p, \varphi}\left(\mathbf{R}_{+}\right)$, there 
exists a unique representation $T_{1}^{(1)} f=h_{1} \in L_{p, \psi^{1-p}}\left(\mathbf{R}_{+}\right)$, satisfying for any $y \in \mathbf{R}_{+}, T_{1}^{(1)} f(y)=$ $h_{1}(y)$.

In view of (32), it follows that $\left\|T_{1}^{(1)} f\right\|_{p, \psi^{1-p}}=\left\|h_{1}\right\|_{p, \psi^{1-p}} \leq M_{1}\|f\|_{p, \varphi}$, and then the operator $T_{1}^{(1)}$ is bounded satisfying

$$
\left\|T_{1}^{(1)}\right\|=\sup _{f(\neq \theta) \in L_{p, \varphi}\left(R_{+}\right)} \frac{\left\|T_{1}^{(1)} f\right\|_{p, \psi^{1-p}}}{\|f\|_{p, \varphi}} \leq M_{1} .
$$

If we define the formal inner product of $T_{1}^{(1)} f$ and $g$ as follows:

$$
\left(T_{1}^{(1)} f, g\right):=\int_{0}^{\infty}\left(\int_{0}^{\frac{1}{y}} \frac{(\min \{x y, 1\})^{\alpha}|\ln x y|^{\beta}}{(\max \{x y, 1\})^{\lambda+\alpha}} f(x) d x\right) g(y) d y,
$$

then we can rewrite Theorem 1 (for $\sigma_{1}=\sigma$ ) as follows.

Theorem 3 If $p>1, \frac{1}{p}+\frac{1}{q}=1, \beta>-1, \sigma>-\alpha$, then the following conditions are equivalent:

(i) There exists a constant $M_{1}$, such that, for any $f(x) \geq 0, f \in L_{p, \varphi}\left(\mathbf{R}_{+}\right),\|f\|_{p, \varphi}>0$, we have the following inequality:

$$
\left\|T_{1}^{(1)} f\right\|_{p, \psi^{1-p}}<M_{1}\|f\|_{p, \varphi}
$$

(ii) There exists a constant $M_{1}$, such that, for any $f(x), g(y) \geq 0, f \in L_{p, \varphi}\left(\mathbf{R}_{+}\right)$, $g \in L_{q, \psi}\left(\mathbf{R}_{+}\right),\|f\|_{p, \varphi},\|g\|_{q, \psi}>0$, we have the following inequality:

$$
\left(T_{1}^{(1)} f, g\right)<M_{1}\|f\|_{p, \varphi}\|g\|_{q, \psi} .
$$

We still have $\left\|T_{1}^{(1)}\right\|=k_{1}(\sigma) \leq M_{1}$.

(b) In view of Corollary $1\left(\mu_{1}=\mu\right)$, for $f \in L_{p, \varphi}\left(\mathbf{R}_{+}\right)$, setting

$$
h_{2}(y):=\int_{0}^{y} \frac{(\min \{x, y\})^{\alpha}|\ln (x / y)|^{\beta}}{(\max \{x, y\})^{\lambda+\alpha}} f(x) d x \quad\left(y \in \mathbf{R}_{+}\right),
$$

by (26), we have

$$
\left\|h_{2}\right\|_{p, \phi^{1-p}}=\left[\int_{0}^{\infty} \phi^{1-p}(y) h_{2}^{p}(y) d y\right]^{\frac{1}{p}}<M_{1}\|f\|_{p, \varphi}<\infty .
$$

Definition 2 Define a Hardy-type integral operator of the first kind with the homogeneous kernel $T_{1}^{(2)}: L_{p, \varphi}\left(\mathbf{R}_{+}\right) \rightarrow L_{p, \phi^{1-p}}\left(\mathbf{R}_{+}\right)$as follows: For any $f \in L_{p, \varphi}\left(\mathbf{R}_{+}\right)$, there exists a unique representation $T_{1}^{(2)} f=h_{2} \in L_{p, \phi^{1-p}}\left(\mathbf{R}_{+}\right)$, satisfying for any $y \in \mathbf{R}_{+}, T_{1}^{(2)} f(y)=h_{2}(y)$.

In view of (35), it follows that $\left\|T_{1}^{(2)} f\right\|_{p, \phi^{1-p}}=\left\|h_{2}\right\|_{p, \phi^{1-p}} \leq M_{1}\|f\|_{p, \varphi}$, and then the operator $T_{1}^{(2)}$ is bounded satisfying

$$
\left\|T_{1}^{(2)}\right\|=\sup _{f(\neq \theta) \in L_{p, \varphi}\left(R_{+}\right)} \frac{\left\|T_{1}^{(2)} f\right\|_{p, \phi^{1-p}}}{\|f\|_{p, \varphi}} \leq M_{1} .
$$


If we define the formal inner product of $T_{1}^{(2)} f$ and $g$ as follows:

$$
\left(T_{1}^{(2)} f, g\right):=\int_{0}^{\infty}\left(\int_{0}^{y} \frac{(\min \{x, y\})^{\alpha}|\ln (x / y)|^{\beta}}{(\max \{x, y\})^{\lambda+\alpha}} f(x) d x\right) g(y) d y,
$$

then we can rewrite Corollary 1 (for $\mu_{1}=\mu$ ) as follows.

Corollary 3 If $p>1, \frac{1}{p}+\frac{1}{q}=1, \beta>-1, \sigma=\lambda-\mu>-\alpha$, then the following conditions are equivalent:

(i) There exists a constant $M_{1}$, such that, for any $f(x) \geq 0, f \in L_{p, \varphi}\left(\mathbf{R}_{+}\right),\|f\|_{p, \varphi}>0$, we have the following inequality:

$$
\left\|T_{1}^{(2)} f\right\|_{p, \phi^{1-p}}<M_{1}\|f\|_{p, \varphi} .
$$

(ii) There exists a constant $M_{1}$, such that, for any $f(x), g(y) \geq 0, f \in L_{p, \varphi}\left(\mathbf{R}_{+}\right), g \in$ $L_{q, \phi}\left(\mathbf{R}_{+}\right),\|f\|_{p, \varphi},\|g\|_{q, \phi}>0$, we have the following inequality:

$$
\left(T_{1}^{(2)} f, g\right)<M_{1}\|f\|_{p, \varphi}\|g\|_{q, \phi} .
$$

We still have $\left\|T_{1}^{(2)}\right\|=k_{1}(\sigma) \leq M_{1}$.

Remark 4 Theorem 3 and Corollary 3 are equivalent.

(c) In view of Theorem $2\left(\sigma_{1}=\sigma\right)$, for $f \in L_{p, \varphi}\left(\mathbf{R}_{+}\right)$, setting

$$
H_{1}(y):=\int_{\frac{1}{y}}^{\infty} \frac{(\min \{x y, 1\})^{\alpha}|\ln x y|^{\beta}}{(\max \{x y, 1\})^{\lambda+\alpha}} f(x) d x \quad\left(y \in \mathbf{R}_{+}\right)
$$

by (28), we have

$$
\left\|H_{1}\right\|_{p, \psi^{1-p}}=\left[\int_{0}^{\infty} \psi^{1-p}(y) H_{1}^{p}(y) d y\right]^{\frac{1}{p}}<M_{2}\|f\|_{p, \varphi}<\infty .
$$

Definition 3 Define a Hardy-type integral operator of the second kind with the nonhomogeneous kernel $T_{2}^{(1)}: L_{p, \varphi}\left(\mathbf{R}_{+}\right) \rightarrow L_{p, \psi} 1-p\left(\mathbf{R}_{+}\right)$as follows: For any $f \in L_{p, \varphi}\left(\mathbf{R}_{+}\right)$, there exists a unique representation $T_{2}^{(1)} f=H_{1} \in L_{p, \psi^{1-p}}\left(\mathbf{R}_{+}\right)$, satisfying for any $y \in \mathbf{R}_{+}, T_{2}^{(1)} f(y)=$ $H_{1}(y)$.

In view of (38), it follows that $\left\|T_{2}^{(1)} f\right\|_{p, \psi 1-p}=\left\|H_{1}\right\|_{p, \psi 1-p} \leq M_{2}\|f\|_{p, \varphi}$, and then the operator $T_{2}^{(1)}$ is bounded satisfying

$$
\left\|T_{2}^{(1)}\right\|=\sup _{f(\neq \theta) \in L_{p, \varphi}\left(R_{+}\right)} \frac{\left\|T_{2}^{(1)} f\right\|_{p, \psi^{1-p}}}{\|f\|_{p, \varphi}} \leq M_{2} .
$$

If we define the formal inner product of $T_{2}^{(1)} f$ and $g$ as follows:

$$
\left(T_{2}^{(1)} f, g\right):=\int_{0}^{\infty}\left(\int_{\frac{1}{y}}^{\infty} \frac{(\min \{x y, 1\})^{\alpha}|\ln x y|^{\beta}}{(\max \{x y, 1\})^{\lambda+\alpha}} f(x) d x\right) g(y) d y,
$$

then we can rewrite Theorem 2 (for $\sigma_{1}=\sigma$ ) as follows. 
Theorem 4 If $p>1, \frac{1}{p}+\frac{1}{q}=1, \beta>-1, \mu=\lambda-\sigma>-\alpha$, then the following conditions are equivalent:

(i) There exists a constant $M_{2}$, such that, for any $f(x) \geq 0, f \in L_{p, \varphi}\left(\mathbf{R}_{+}\right),\|f\|_{p, \varphi}>0$, we have the following inequality:

$$
\left\|T_{2}^{(1)} f\right\|_{p, \psi^{1-p}}<M_{2}\|f\|_{p, \varphi}
$$

(ii) There exists a constant $M_{2}$, such that, for any $f(x), g(y) \geq 0, f \in L_{p, \varphi}\left(\mathbf{R}_{+}\right)$,

$g \in L_{q, \psi}\left(\mathbf{R}_{+}\right),\|f\|_{p, \varphi},\|g\|_{q, \psi}>0$, we have the following inequality:

$$
\left(T_{2}^{(1)} f, g\right)<M_{2}\|f\|_{p, \varphi}\|g\|_{q, \psi} \cdot
$$

We still have $\left\|T_{2}^{(1)}\right\|=k_{1}(\mu) \leq M_{2}$.

(d) In view of Corollary $2\left(\mu_{1}=\mu\right)$, for $f \in L_{p, \varphi}\left(\mathbf{R}_{+}\right)$, setting

$$
H_{2}(y):=\int_{y}^{\infty} \frac{(\min \{x, y\})^{\alpha}|\ln (x / y)|^{\beta}}{(\max \{x, y\})^{\lambda+\alpha}} f(x) d x \quad\left(y \in \mathbf{R}_{+}\right),
$$

by (30), we have

$$
\left\|H_{2}\right\|_{p, \phi^{1-p}}=\left[\int_{0}^{\infty} \phi^{1-p}(y) H_{2}^{p}(y) d y\right]^{\frac{1}{p}}<M_{2}\|f\|_{p, \varphi}<\infty .
$$

Definition 4 Define a Hardy-type integral operator of the second kind with the homogeneous kernel $T_{2}^{(2)}: L_{p, \varphi}\left(\mathbf{R}_{+}\right) \rightarrow L_{p, \phi^{1}-p}\left(\mathbf{R}_{+}\right)$as follows: For any $f \in L_{p, \varphi}\left(\mathbf{R}_{+}\right)$, there exists a unique representation $T_{2}^{(2)} f=H_{2} \in L_{p, \phi^{1-p}}\left(\mathbf{R}_{+}\right)$, satisfying for any $y \in \mathbf{R}_{+}, T_{2}^{(2)} f(y)=H_{2}(y)$.

In view of (41), it follows that $\left\|T_{2}^{(2)} f\right\|_{p, \phi^{1}-p}=\left\|H_{2}\right\|_{p, \phi^{1-p}} \leq M_{2}\|f\|_{p, \varphi}$, and then the operator $T_{2}^{(2)}$ is bounded satisfying

$$
\left\|T_{2}^{(2)}\right\|=\sup _{f(\neq \theta) \in L_{p, \varphi}\left(R_{+}\right)} \frac{\left\|T_{2}^{(2)} f\right\|_{p, \phi^{1-p}}}{\|f\|_{p, \varphi}} \leq M_{2} .
$$

If we define the formal inner product of $T_{1}^{(2)} f$ and $g$ as follows:

$$
\left(T_{2}^{(2)} f, g\right):=\int_{0}^{\infty}\left(\int_{y}^{\infty} \frac{(\min \{x, y\})^{\alpha}|\ln (x / y)|^{\beta}}{(\max \{x, y\})^{\lambda+\alpha}} f(x) d x\right) g(y) d y
$$

then we can rewrite Corollary 2 (for $\mu_{1}=\mu$ ) as follows.

Corollary 4 If $p>1, \frac{1}{p}+\frac{1}{q}=1, \beta>-1, \mu=\lambda-\sigma>-\alpha$, then the following conditions are equivalent:

(i) There exists a constant $M_{2}$, such that, for any $f(x) \geq 0, f \in L_{p, \varphi}\left(\mathbf{R}_{+}\right),\|f\|_{p, \varphi}>0$, we have the following inequality:

$$
\left\|T_{2}^{(2)} f\right\|_{p, \phi^{1-p}}<M_{2}\|f\|_{p, \varphi}
$$


(ii) There exists a constant $M_{2}$, such that, for any $f(x), g(y) \geq 0, f \in L_{p, \varphi}\left(\mathbf{R}_{+}\right)$, $g \in L_{q, \phi}\left(\mathbf{R}_{+}\right),\|f\|_{p, \varphi},\|g\|_{q, \phi}>0$, we have the following inequality:

$$
\left(T_{2}^{(2)} f, g\right)<M_{2}\|f\|_{p, \varphi}\|g\|_{q, \phi}
$$

We still have $\left\|T_{2}^{(2)}\right\|=k_{1}(\mu) \leq M_{2}$.

Remark 5 Theorem 4 and Corollary 4 are equivalent.

\section{Conclusions}

In this paper, by means of real analysis and weight functions a few equivalent conditions of two kinds of Hardy-type integral inequalities with the non-homogeneous kernel and parameters are obtained by Theorem 1, 2. The constant factors related to the gamma function are proved to be the best possible. We also consider the operator expressions in Theorem 3, 4. The dependent cases of homogeneous kernel are assumed by Corollary 14. The method of weight functions is very important, it is the key to help us proving the main inequalities with the best possible constant factor. The lemmas provide an extensive account of this type of inequalities.

\section{Acknowledgements}

This work is supported by the National Natural Science Foundation (No. 11401113), and Appropriative Researching Fund for Professors and Doctors, Guangdong University of Education (No. 2015ARF25). We are grateful for their help.

Competing interests

The authors declare that they have no competing interests.

Authors' contributions

BY carried out the mathematical studies, participated in the sequence alignment and drafted the manuscript. JL participated in the design of the study and performed the numerical analysis. All authors read and approved the final manuscript.

\section{Publisher's Note}

Springer Nature remains neutral with regard to jurisdictional claims in published maps and institutional affiliations.

Received: 29 March 2017 Accepted: 15 May 2017 Published online: 07 June 2017

\section{References}

1. Schur, I: Bernerkungen sur Theorie der beschrankten Billnearformen mit unendlich vielen Veranderlichen. J. Math. $140,1-28(1911)$

2. Hardy, GH: Note on a theorem of Hilbert concerning series of positive terms. Proceedings London Math. Soc. 23(2), (1925), Records of Proc. XLV-XLVI

3. Hardy, GH, Littlewood, JE, Pólya, G: Inequalities. Cambridge University Press, Cambridge (1934)

4. Mitrinović, DS, Pečarić, JE, Fink, AM: Inequalities Involving Functions and Their Integrals and Derivatives. Kluwer Academic, Boston (1991)

5. Yang, BC: On Hilbert's integral inequality. J. Math. Anal. Appl. 220, 778-785 (1998)

6. Yang, BC: A note on Hilbert's integral inequality. Chin. Q. J. Math. 13(4), 83-86 (1998)

7. Yang, BC: On an extension of Hilbert's integral inequality with some parameters. Aust. J. Math. Anal. Appl. 1(1), Article ID 11 (2004)

8. Yang, BC, Brnetić, I, Krnić, M, Pečarić, JE: Generalization of Hilbert and Hardy-Hilbert integral inequalities. Math. Inequal. Appl. 8(2), 259-272 (2005)

9. Krnić, M, Pečarić, JE: Hilbert's inequalities and their reverses. Publ. Math. (Debr.) 67(3-4), 315-331 (2005)

10. Hong, Y: On Hardy-Hilbert integral inequalities with some parameters. J. Inequal. Pure Appl. Math. 6(4), Article ID 92 (2005)

11. Arpad, B, Choonghong, O: Best constant for certain multi linear integral operator. J. Inequal. Appl. 2006, Article ID $28582(2006)$

12. Li, YJ, He, B: On inequalities of Hilbert's type. Bull. Aust. Math. Soc. 76(1), 1-13 (2007)

13. Zhong, WY, Yang, BC: On multiple Hardy-Hilbert's integral inequality with kernel. J. Inequal. Appl. 2007, Article ID 27962 (2007)

14. Xu, JS: Hardy-Hilbert's inequalities with two parameters. Adv. Math. 36(2), 63-76 (2007)

15. Yang, BC: The Norm of Operator and Hilbert-Type Inequalities. Science Press, Beijing (2009) 
16. Yang, BC: Hilbert-Type Integral Inequalities. Bentham Science Publishers Ltd., Sharjah (2009)

17. Yang, BC: On Hilbert-type integral inequalities and their operator expressions. J. Guangaong Univ. Educ. 33(5), 1-17 (2013)

18. Hong, Y: On the structure character of Hilbert's type integral inequality with homogeneous kernel and applications. J. Jilin Univ. Sci. Ed. 55(2), 189-194 (2017)

19. Wang, ZQ, Guo, DR: Introduction to Special Functions. Science Press, Beijing (1979)

20. Kuang, JC: Real and Functional Analysis (Continuation) (Second Volume). Higher Education Press, Beijing (2015)

21. Kuang, JC: Applied Inequalities. Shangdong Science and Technology Press, Jinan (2004)

Submit your manuscript to a SpringerOpen ${ }^{\odot}$ journal and benefit from:

- Convenient online submission

- Rigorous peer review

- Immediate publication on acceptance

- Open access: articles freely available online

- High visibility within the field

- Retaining the copyright to your article

Submit your next manuscript at $>$ springeropen.com 